\title{
The Effect of Alcoholic Extract of Persian Gulf Chiton (lamyi) Shell on Angiogenesis in Chick Chorioallantoic Membrane
}

\author{
Toktam Javan-Jaafari-Bojnourdi ${ }^{1}$; Javad Baharara ${ }^{1, *}$ \\ ${ }^{1}$ Research Center for Animal Development Applied Biology, Mashhad Branch, Islamic Azad University, Mashhad, Iran \\ *Corresponding author: Javad Baharara, Research Center for Animal Development Applied Biology, Mashhad Branch, Islamic Azad University, Mashhad, Iran., E-mail: baharara@ \\ yahoo.com
}

Received: February 22, 2014; Accepted: April 2, 2014

\begin{abstract}
Background: Inhibition of angiogenesis is a major target in control and treatment of many disorders depend of angiogenesis. Chiton is a marine mollusk and it can be useful for treatment of diseases because of exist many chemical compositions in its. We investigated the effects of shell extract of chiton on angiogenesis in chorioallantoic membrane.

Materials and Methods: In this experimental study, we used 30 Ross fertilized eggs that were divided into 3 random groups: control, sham-exposed (treated by dimethyle sulfoxide: DMSO) and experimental (treated with shell extract). In 2nd day, a window was opened on eggs in the sterile condition. Later, in 8nd day, a gelatin sponge appeared on chorioallantoic membrane and was soaked with $10 \mu \mathrm{L}$ extract in treatment group and $10 \mu \mathrm{L}$ DMSO in the sham-exposed group. In 12nd day, chorioallantoic membranes (CAMs) were photographed by research photo stereomicroscope in all cases. The numbers and lengths of vessels around the sponges were measured and compared with each other by SPSS-16 software and ANOVA $(\mathrm{p}<0.05)$.

Results: The mean number of vessels $(10.34 \pm 1.85)$ and length of vessels $(13.12 \pm 2.04 \mathrm{~mm})$ in the control group and mean number of vessels $(9.97 \pm 1.38)$ and length of vessels $(13.42 \pm 1.08 \mathrm{~mm}$ ) in sham-exposed group was not any significant differences ( $p>0.05)$. There was a significant decrease in mean number of vessels $(6.06 \pm 1.36)$ and length of vessels $(9.76 \pm 1.21 \mathrm{~mm})$ in experimental group.

Conclusion: It seems shell extract of Chiton lamyican decreases the number and length of vessels around treated area so it can be used as inhibitory agent in treatment of angiogenesis dependent disease.
\end{abstract}

Keywords:Angiogenesis; Chorioallantoic Membrane; Shell

\section{Introduction}

The ocean, which covers around 70\% of the Earth's surface, is a rich source of natural resources that contains nearly $80 \%$ of all the varieties of life on our planet. During the past 30 years, thousands of new compounds with different biological activities varying from anticancer to antiviral have been isolated from marine sources [1]. Biologically active molecules isolated from marine flora and fauna have applications in pharmaceuticals, nutritional supplements, cosmetics, agrochemicals, molecular probes, enzymes and fine chemicals [2]. Many marine derived natural products and their analogues have been reported to show antiangiogenic activities [3].

Angiogenesis is a complex biological process defined as the formation of new blood vessels from preexisting vessels [4]. Angiogenesis is important in physiological processes such as wound healing, growth and development of organs and also menstrual cycles [5]. Angiogenesis not only plays an important role in physiological processes but is also involved in initiating and promoting several diseases such as cancer. As a hallmark of cancer, angiogenesis is considered as a powerful target to suppress tumor growth and metastasis [3]. The chick embryo cho- rioallantoic membrane (CAM) model is an extra embryonic membrane that is commonly used in vivo to study both angiogenesis and anti angiogenesis. An angiogenic response occurs 72-96 $\mathrm{h}$ after stimulation in the form of increased vessel density around the implant, with the vessels radially converging toward the center like spokes in a wheel. Conversely, when an angiostatic compound is tested, the vessels become less dense around the implant and even disappear [6].

Chitons (class polyplacophora) are crawling marine mollusks protected by eight dorsal shell plates made of aragonite [7]. They found worldwide in all seas with more than 940 extant and 430 fossil species which extend to the late Cambrian. Chitons are one of the earliest diverging groups of living mollusks and are often referred to as "living fossils" since their body plan has not significantly changed for over 300 million years [8]. Chitons can be considerable because of exist chitin and chitosan composition in shell of polyplacophora [9].

Low molecular weight chitosans or oligochitosan might be useful in preventing tumor growth [10]. Also indicated chitooligosaccharides (CHOS) (that obtained 
from hydrolysis chitosan) inhibit angiogenesis in vivo and in vitro [11]. With regard to the importance of use natural product in treatment of disease; in this research we investigated anti-angiogenesis effect of Chiton lamyishell extract on chorioallantoic membrane; that has not been studied so far.

\section{Materials and Methods}

This experimental study had been done in research Center of Animal Development Applied Biology of Mashhad Islamic Azad University in 2013. Required chitons were collected from Persian Gulf coasts (Chabahar) then were identified and prepared extraction with methanol solvent. After filtered the extracts, were using a rotary evaporator (vacuum rotary evaporator) concentrated in reduced pressure and then transferred into clean Petri dish were dried in an oven at $45^{\circ} \mathrm{C}$, then obtained extract maintained in $-20^{\circ} \mathrm{C}$.For antiangiogenic effect, 30 fertilized eggs was purchased from Toos company of Mashhad, then were distributed randomly into 3 groups including the control group, the sham exposed (treated with DMSO solvent), and the experimental group (treated with $10 \mu \mathrm{L}$ chiton shell extract). The fertilized eggs were placed at the incubation devise (Digital, Korea) which rotated automatically at the temperature of $38^{\circ} \mathrm{C}$ and relative humidity of $60-65 \%$. In the second day of incubation, at the completely sterilized condition, which was created by laminar hood (Telstar, Spain), a part of the egg shells was removed and a window was made at one side of the eggs, which was closed by lamel and sterilized paraffin (Italia).

Afterwards, the eggs were returned to the incubator and manual rotation was performed twice a day for natural development of the chicks. Since the chorioallantoic membrane of the chicks starts its creation from the 5 th day of incubation and in the 8th day occupies more than half of the eggs and also because in this day the heart is divided and the vein and the artery separation happens, the treatment of the vessel network at the 8th day can be noted [12].

Therefore, in 8th day of incubation, the window of the shells was removed as completely sterilized condition and a gelatin sponge containing the albumen and agar solution in normal saline (with equal ratio) with $200 \mu \mathrm{L}$ of streptomycin penicillin (USA, GIBCO) which was prepared freshly at sterilized condition, were placed on the chorioallantoic membrane at $4 \times 4 \times 1 \mathrm{~mm}^{3}$. In the samples treated with shell extract, $10 \mu \mathrm{L}$ of extract and in shamexposed samples $10 \mu \mathrm{L}$ of DMSO was added to the gelatin sponge and then the places of the windows were again covered and the eggs were returned to the incubator. In 12th day of incubation, all cases were photographed by research photo stereomicroscope (Ziess, Germany) and take appropriate photos (Figure 1). Variables include the number and length of blood vessels that for all samples was measured around gelatin sponge. As chorioallantoic membrane is an anatomic disk like with $400 \mu \mathrm{m}$ diameter, so all blood vessels around gelatin sponge are countable. Quantitative data were obtained using SPSS-16 and ANOVA, independent t-test with significance level of $\mathrm{p}<0.05$, analysis and post hoc Tukey test was performed in cases of need.

\section{Results}

Comparing mean number of vessels $(10.34 \pm 1.85)$ and length of vessels $(13.12 \pm 2.04 \mathrm{~mm})$ in the control group with the number of vessels $(9.97 \pm 1.38)$ and length of vessels $(13.42 \pm 1.08 \mathrm{~mm})$ in sham-exposed group did not show any significant differences. There was a significant decrease in mean number of vessels $(6.06 \pm 1.36)$ and length of vessels $(9.76 \pm 1.21 \mathrm{~mm})$ in experimental group $(\mathrm{p}<0.001)$. It's indicated shell extract of Chiton lamyihave anti angiogenesis effect on chorioallantoic membrane (Figure 2).
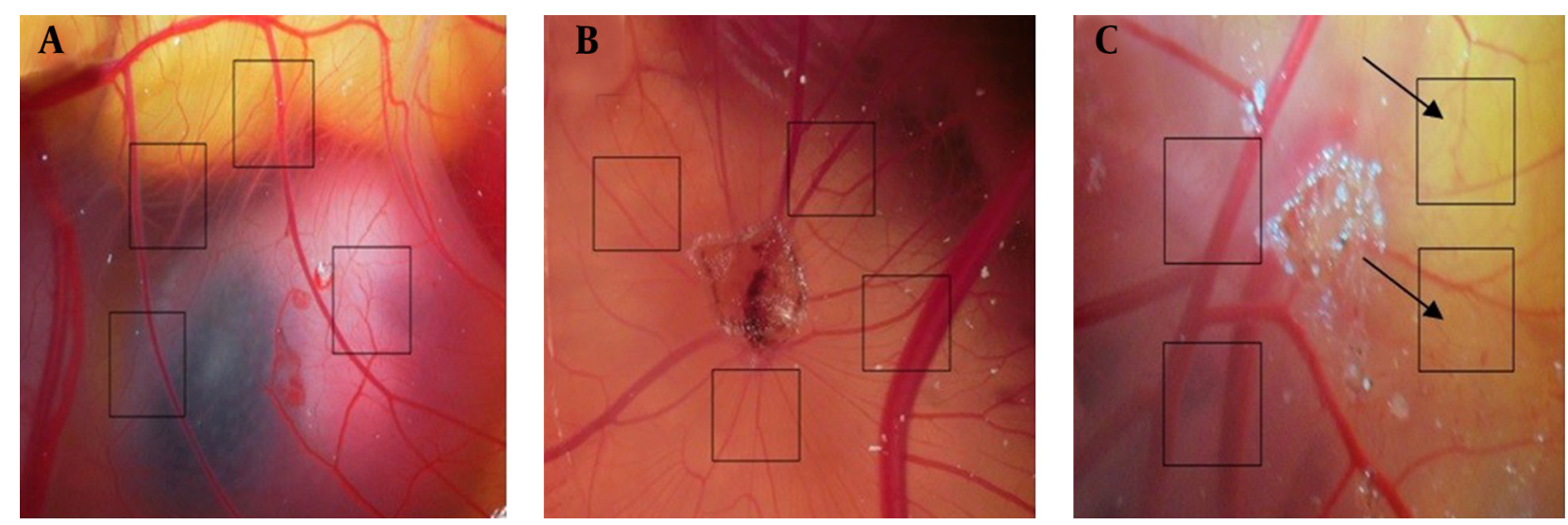

Figure 1. Images of research photo stereomicroscope from different test groups, A- Control sample, B- Sham-exposed sample, C- Experimental sample Squares show counted places by image J software. The arrows show a considerable decrease the number of vessels in the experimental group. 

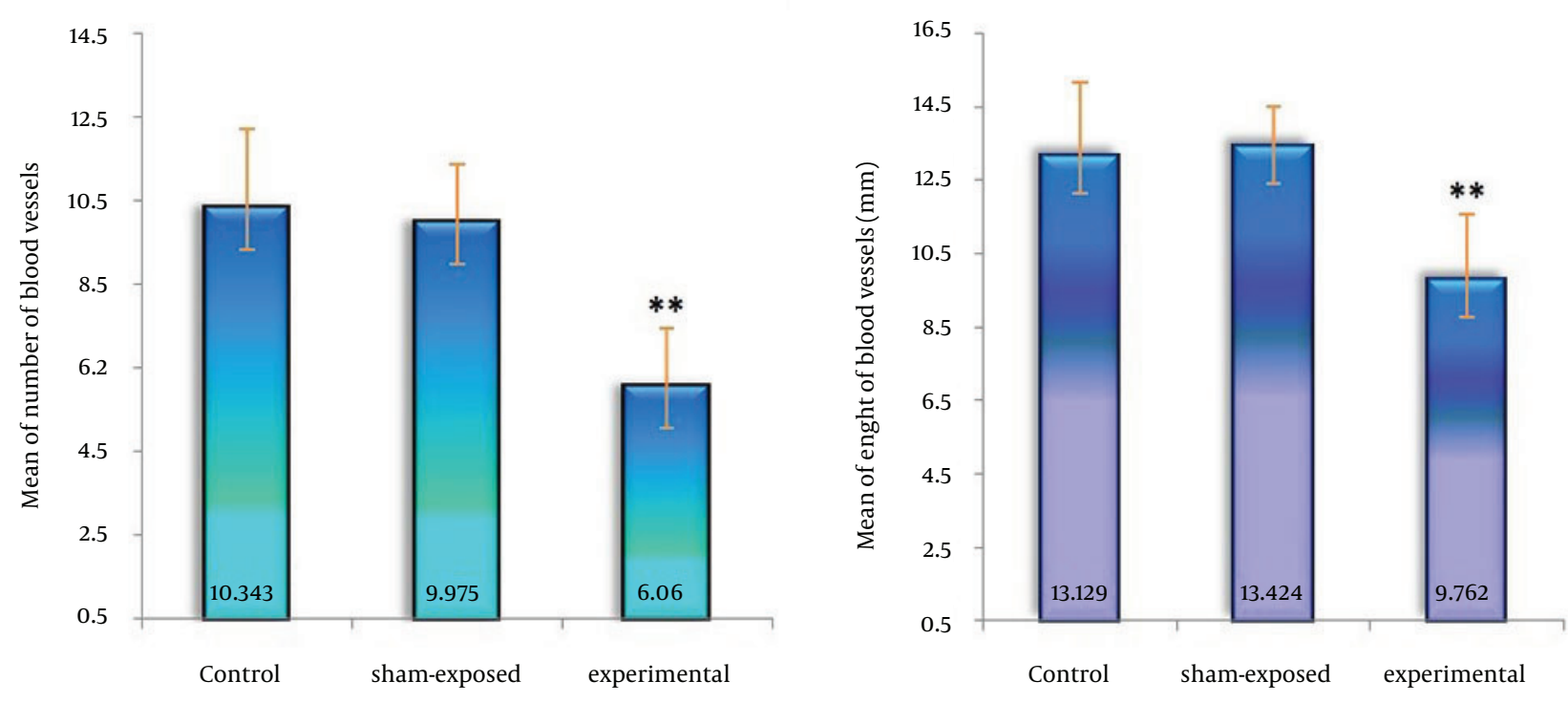

Figure 2. Comparison of average of number and length of vessels in control, sham-exposed and experimental groups. ${ }^{* *} \mathrm{p}<0.001$

\section{Discussion}

According to the results of this study, $10 \mu \mathrm{L}$ of alcoholic extract of chiton shell, reduce significantly the number and length of vessel branching in the chick chorioallantoic membrane in treated group in compare with the control. It can be concluded that this extract has anti angiogenesis properties and could be considered as a suitable agent in cancer treatment in future. Much research has been done concerned with the effects of natural products on angiogenesis in CAM model. Namvar et al. studied the effects of the methanolic extract of Sargassum seaweed on angiogenesis. Their results showed that Sargassum seaweed extract can reduce the length and number of blood vessels in the CAM model [12]. Also Ganesan et al. showed that the siphonaxanthin is derived from green algae can inhibit angiogenesis in ex vivo and in vitro [13]. Also Qianming et al. studied the effects of GFP08 a kind of polysaccharide, which is derived from Grateloupia filicina (brown seaweed) on angiogenesis in CAM. The results showed that this polysaccharide in a dosage dependent manner decreased the formation of the new blood vessels [14]. We have used this model for study anti angiogenesis effect of shell extract of chiton. Anti angiogenesis effects of chitons have not been studied so far. According to one research organic matrix composition of chiton Acanthopleura villantii shell were investigated: the chitin protein ratio is 6 to 9 , which is more than a hundred times greater than the amount that is found in the shells of other mollusks [15].

Wu et al. showed effects of different concentrations of chitooligosaccharides on chorioallantoic membrane, that upon which causes reduce the formation and growth of vessels in the CAM model [16]. These results are consistent with our results in this research, because of the presence of chitin in the shell of chiton and its anti angiogenesis effect in CAM model. Zhang and Kim, for the first time, investigated effects of marine chitooligosaccharides on the activation and expression of matrix metalloproteinase 2 in human skin fibroblasts and showed that marine chitooligosaccharides inhibit expression and activity of matrix metalloproteinase 2 [17]. Because of presence chitin and chitosan in molluska $[18,19]$ and anti angiogenesis effects of chitooligosaccharides [16], probably presence chitin and chitosan in chiton shell is one of the reasons for its decreasing effect on angiogenesis.

Many researchers have been done about anti angiogenesis effects of natural substances extracted from different species of molluska. Dolastatin is a cytotoxic peptide is derived from the genus Dolabella auricularia and it is an anti cancer agent. Dolastatin inhibit cell proliferation and intracellular death (apoptosis) is induced in many cancer cell lines [20]. Kahalalides is a family of peptides isolated from the sacoglossan mollusk Elysia rufescens. Among these, Kahalalide $\mathrm{F}$ is a dehydroaminobutyric acid containing peptide. This is known to exhibit interesting antitumor activity. It has been observed that Kahalalide F induces disturbances in lysosomal function that might lead to intracellular acidification and cell death. Extensive experiments show that this compound has a potent activity on non small cell lung cancer, melanoma, androgen independent prostate cancer, hepatocellular carcinoma, colon cancer and breast cancer [21].

The results show that shell extract of chiton has an inhibitory effect on angiogenesis and decrease the process of angiogenesis in chick chorioallantoic membrane. 
The results of this research could be start for another research on chiton and its active metabolites. So it can be used as an inhibitory agent in control of angiogenesis dependent disease.

\section{Acknowledgements}

The authors would like to thank respectful researchers at Research Center for Animal Development Applied Biology, Islamic Azad University of Mashhad who collaborated in done this project (Code number: 11130517912015 ).

\section{Authors' Contributions}

All authors had equal role in design, work, statistical analysis and manuscript writing.

\section{Conflict of Interest}

The authors declare no conflict of interest.

\section{Funding/Support}

Research Center for Animal Development Applied Biology, Mashhad Branch, Islamic Azad University.

\section{References}

1. Abraham I, El Sayed K, Chen ZS, Guo H. Current status on marine products with reversal effect on cancer multidrug resistance. MarDrugs. 2012;10(10):2312-21.

2. Thakur AN, Thakur NL, Indap MM, Pandit RA, Datar VV, Muller WE. Antiangiogenic, antimicrobial, and cytotoxic potential of sponge-associated bacteria. Mar Biotechnol (NY). 2005;7(3):245-52.

3. Wang YQ, Miao ZH. Marine-derived angiogenesis inhibitors for cancer therapy. Mar Drugs. 2013;11(3):903-33.

4. Le Guillou-Buffello D, Chesneau C, Gindre M, Hélary G, Laugier $\mathrm{P}$, Migonney V. Inhibition of angiogenesis in vitro with soluble copolymers monitored with a quartz crystal resonator. Irbm. 2010;31(5-6):271-9.

5. Mousavi M, Baharara J, Zafar-Balanezhad S, Shahrokh-Abadi K. Effect of Saffron aqua extract on angiogenesis in chick chorioalantoic membrane. Zahedan J Res Med Sci. 2014;16(3):55-8.

6. Wang S, Zheng Z, Weng Y, Yu Y, Zhang D, Fan W, et al. Angiogenesis and anti-angiogenesis activity of Chinese medicinal herbal extracts. Life Sci. 2004;74(20):2467-78.
7. Connors MJ, Ehrlich H, Hog M, Godeffroy C, Araya S, Kallai I, et al. Three-dimensional structure of the shell plate assembly of the chiton Tonicella marmorea and its biomechanical consequences. J Struct Biol. 2012;177(2):314-28.

8. Peters W. Occurrence of chitin in mollusca. Comparative Biochemistry and Physiology Part B: Comparative Biochemistry. 1972; 41(3):541-50.

9. Maeda Y, Kimura Y. Antitumor effects of various low-molecularweight chitosans are due to increased natural killer activity of intestinal intraepithelial lymphocytes in sarcoma 180-bearing mice. J Nutr. 2004;134(4):945-50.

10. Wu H, Yao Z, Bai X, Du Y, Ma X. Chitooligosaccharides inhibit nitric oxide mediated migration of endothelial cells in vitro and tumor angiogenesis in vivo. Carbohydrate Polymers. 2010;82(3):927-32.

11. Baharara J, Ashraf A, Balanejad S, Samareh Mosavi S. [The inhibitory effect of low frequency electromagnetic field $(50 \mathrm{~Hz})$ on angiogenesis in chorioallantoic membrane of chick] Persian. Zahedan J Res Med Sci. 2010;12(2):12-8.

12. Namvar F, Mohamad R, Baharara J, Zafar-Balanejad S, Fargahi F, Rahman HS. Antioxidant, antiproliferative, and antiangiogenesis effects of polyphenol-rich seaweed (Sargassum muticum). Biomed Res Int. 2013;2013:604787.

13. Ganesan P, Matsubara K, Ohkubo T, Tanaka Y, Noda K, Sugawara T, et al. Anti-angiogenic effect of siphonaxanthin from green alga, Codium fragile. Phytomedicine. 2010;17(14):1140-4.

14. Yu Q, Yan J, Wang S, Ji L, Ding K, Vella C, et al. Antiangiogenic effects of GFP08, an agaran-type polysaccharide isolated from Grateloupia filicina. Glycobiology. 2012;22(10):1343-52.

15. Furuhashi T, Beran A, Blazso M, Czegeny Z, Schwarzinger C, Steiner G. Pyrolysis GC/MS and IR spectroscopy in chitin analysis of molluscan shells. Biosci Biotechnol Biochem. 2009;73(1):93-103.

16. Wu H, Aam BB, Wang W, Norberg AL, Sorlie M, Eijsink VG, et al. Inhibition of angiogenesis by chitooligosaccharides with specific degrees of acetylation and polymerization. Carbohydr Polym. 2012;89(2):511-8.

17. Zhang C, Kim SK. Matrix metalloproteinase inhibitors (MMPIs) from marine natural products: the current situation and future prospects. Mar Drugs. 2009;7(2):71-84.

18. Sagheer FA, Al-Sughayer MA, Muslim S, Elsabee MZ. Extraction and characterization of chitin and chitosan from marine sources in Arabian Gulf. Carbohydr Polym. 2009;77(2):410-9.

19. Abdou ES, Nagy KS, Elsabee MZ. Extraction and characterization of chitin and chitosan from local sources. Bioresour Technol. 2008;99(5):1359-67.

20. Rajeev K, Xu Z. Biomedical Compounds from Marine organisms. Marine Drugs. 2004;2(3):123-46.

21. Malaker A, Ishtiyaq Ahmad S. Therapeutic potency of anticancer peptides derived from marine organism. Int J Eng Appl Sci. 2013;2(3):82-94. 